\title{
THE THIXOTROPY AND DILATANCY OF A MARINE SOIL
}

\author{
By Garth Chapman, M.A. \\ From the Department of Zoology, Queen Mary College, London
}

(Text-figs. I-6)

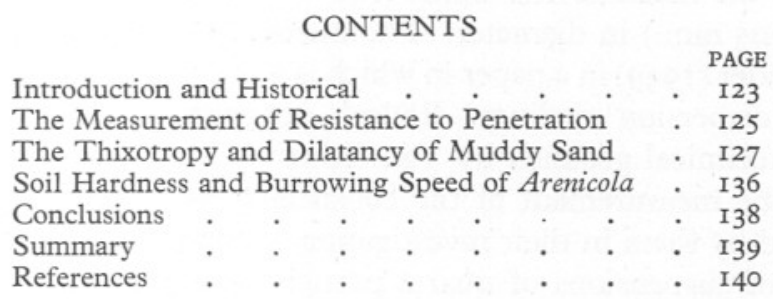

\section{INTRODUCTION AND HISTORICAL}

In a previous paper (Chapman \& Newell, 1947) attention was called to the importance to their inhabitants of the thixotropic ${ }^{\star}$ and dilatant properties of marine soils. It will be recalled that the property of dilatancy, shown by the whitening of wet sand under the footstep, was first described by Osborne Reynolds (1885), and has subsequently been investigated chiefly by Freundlich and his collaborators (Freundlich, 1935; Freundlich \& Jones, 1936; Freundlich \& Röder, 1938). Reynolds considered that the close packing of particles in a liquid medium was altered by an applied force so that the interstitial volume was increased and more liquid was drawn from the periphery into the dilatant mixture. By these changes the dilatant mass becomes harder and more resistant to shear. Freundlich \& Röder described the relation between thixotropy and dilatancy. They considered that thixotropy could be described as a reduction in resistance with increased rate of shear as opposed to dilatancy, in which increased shearing force brings about an increased resistance. Thixo-

\footnotetext{
* The term thixotropy was originally used by Peterfi and Freundlich for the isothermal reversible sol-gel transformation shown by some colloidal solutions, but it has now come to be applied more generally and is used to denote a system which shows a decrease in viscosity upon agitation or a decreased resistance to shear when the rate of shear increases. For example, a suspension of fullers' earth in water liquefies on being shaken or on being stirred, but sets in a few seconds when the agitation stops. Many paints are thixotropic and flow easily and smoothly when they are being applied (whilst they are subject to shearing forces) but their viscosity increases enough when brushing stops for them not to 'run'. Some interesting aspects of the subject are dealt with in articles by Pryce-Jones (1946) and Boswell (1948).
} 
tropic properties are shown by a variety of substances among which are clays whose particles are anisotropic, being rod-like or plate-like in shape. The range of concentration of solid particles to liquid is also important in influencing the behaviour of the suspension to shearing forces, and in their work on quartz particles ( $\mathrm{I}-5 \mu$ in diameter) in water Freundlich \& Röder stated that dilatancy occurred only in the range $42-45 \%$ by volume of the solid. Below $42 \%$ the mixture remained fluid however rapidly it was stirred, whereas above $45 \%$ it remained compact. They described a dilatancy viscometer for use with solid suspensions by means of which they investigated the thixotropic and dilatant properties of a number of mixtures. It was admitted, however, that the machine was difficult to use with suspensions of particles above $50 \mu(0.05 \mathrm{~mm}$.) in diameter. The dilatancy viscometer was described in detail by Röder (1939) in a paper in which is also set out the relation between particles and dispersion medium. Röder's apparatus and results have been criticized on technical grounds by Van Selms \& Kruyt (1943), and another machine for the measurement of the consistency of plastic suspensions has been described by them in their investigation of the effect of a third phase on the rheology of suspensions of quartz particles and glass spheres in carbon tetrachloride (Kruyt \& Van Selms, I943a, b). It is not suitable for use with marine soils.

Freundlich \& Juliusburger (1935) described an investigation into the thixotropy of quicksands and came to the conclusion that, whereas some quicksands have a larger clay content than neighbouring ordinary sands, this cannot be taken as a generalization and that other factors must be taken into account.

The soil of the Whitstable Flats. Clays, in general, are notably thixotropic, and it may be that even a small amount of clay is sufficient to alter the properties of suspensions of particles of larger size. Sand from the Flats at Whitstable in which Arenicola marina is so abundant can be said to possess quicksand or thixotropic properties, although to a much lesser extent than those soils which are recognized as true quicksands. The soil of the Flats is reasonably uniform for a distance of about $400 \mathrm{yd}$. between tidemarks and is characterized by having a sandy-coloured upper layer of about $I$ in. in depth and a deeper layer of 6-I2 in. in depth, black in colour but similar in particlesize composition. On exposure to the air this black sand becomes identical in appearance with that of the uppermost layer, the two layers being similar in all features except in the chemical composition of the iron compounds which they contain. A preliminary investigation showed that the soil consists largely of 'fine sand', as defined by Robinson (1932), of particle size between 0.2 and $0.02 \mathrm{~mm}$. diameter with an admixture of I-3\% of 'silt and clay' of particle size less than $0.02 \mathrm{~mm}$. diameter. Eighty per cent. of the sand passes a I00mesh sieve and therefore has a particle diameter of less than $0.12 \mathrm{~mm}$. which, although above the upper limit of $0.05 \mathrm{~mm}$. diameter given by Freundlich \& Röder as suitable for use in the dilatancy viscometer, is not so widely dif- 
ferent from their figure as to suggest that useful results might not be obtained by the use of their machine.

The interest in the present inquiry lies in the relation of the physical properties of marine soils to the habits of the animals which inhabit them. Some of the properties which appear to be of importance and which have been studied are the resistance to penetration, the thixotropic properties and their variations with changes of composition of the soil.

I am grateful for help at various times to Professor P. G. H. Boswell, F.R.S., Professor A. J. Grove, Dr G. E. Newell and Dr G. W. Scott-Blair.

\section{The Measurement of Resistance to Penetration}

For the easy testing of marine soils in situ the apparatus described by Chapman \& Newell (I947) was used. This penetrometer was modified by the provision of a scale by which readings in the field were made easier to take. A diagram of the modified instrument is given in Fig. I, from which it can be seen that when the slider or collar $(C)$ is held in the hand, and the disk $(D)$ placed upon the sand, pressures varying from zero upwards can be obtained. By calibrating the penetrometer against weights counterpoised on a balance the thrust exerted on the sand by the disk can be measured. A series of springs of different gauge steel wire give the instrument a considerable range. A graph of weight counterpoised against scale reading was made for each spring. This rather crude method of calibration had the advantage that the instrument was manipulated in the way in which it was used in the field when measurements were being made.

In use the instrument was held as nearly vertical as possible and was pressed gently into the soil, the reading on the scale being then noted. Ten or more readings were always made and a mean calculated, the pressure equivalent to the scale reading being then read off from the graph. The hardness of the soil is here expressed as grams per square centimetre necessary for penetration. It was fairly easy, with practice, to obtain a 'good' or close set of figures for any particular locality, but in order to do so it was necessary on occasions to discard readings, for example, when it was clear that the penetrating disk had struck a larger stone or had penetrated a worm burrow.

The measurements made with the penetrometer are correlated in this paper with other features of the soil such as water content or the burrowing speed of Arenicola. Accordingly, the results are set out in conjunction with details of the features with which a connexion was being sought and the results of the tests (expressed always as $\mathrm{g} . / \mathrm{cm} .{ }^{2}$ necessary for the penetration of a disk of area $0.125 \mathrm{~cm} .{ }^{2}$ to a depth of $3 \mathrm{~cm}$.) will be found in the section dealing with the burrowing speed of the lugworm (p. 136). But, to anticipate briefly, both the hardness of the soil and the time taken by the lugworms to burrow was found to increase as the water content decreased. 
Penetrometer

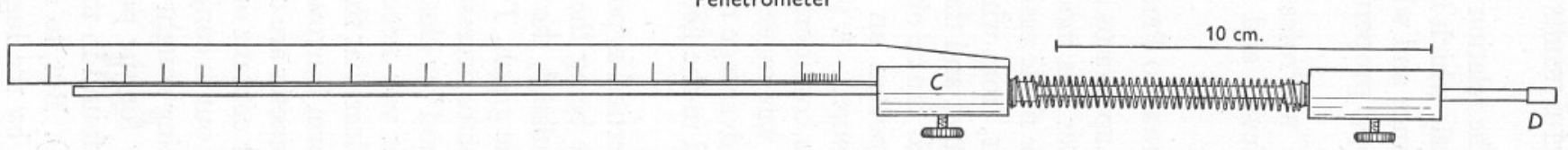

Fig. I. The modified penetrometer. $C$, slider or collar. $D$, penetrating disk. 


\section{The Thixotropy and Dilatancy of Muddy Sand}

One striking feature of the soil of the Flats is the difference between its physical properties when wet and when dry. For example, if water is slowly added to a sample of dry sand it becomes a darker brown as it is wetted. It is hard when touched with a glass rod. Small portions, made up of adhering particles, can be broken off. It does not appear to flow when it is tapped. In fact, it behaves almost as a friable solid.

As more water is added the material 'settles' when the vessel containing it is agitated so that it has a level, shiny but not plane, surface. There is, therefore, sufficient water to appear at the interstices of the superficial particles. When the mass is prodded with a glass rod the colour becomes lighter and the appearance no longer shiny but dull or matt. Its resistance to penetration increases as the rod is pushed in.

The addition of still more water causes the surface to become plane; there is a layer of water unbroken by particles. No lightening of the colour occurs when the mass is prodded nor does the surface become matt in appearance. Its resistance to prodding is smaller and does not increase as distortion occurs.

In other words, the mixture of sand and water apparently shows a variety of properties ranging from those of a dilatant to those of a thixotropic material, according to the proportions in which the sand and water are mixed.

An attempt was therefore made to obtain some more exact measurements of the thixotropic and dilatant properties of the sand and to correlate these with the mechanical analysis and water content. Tests were made in the dilatancy viscometer of Freundlich \& Röder which is described below. Before being used the sand was passed through a $2 \mathrm{~mm}$. round-holed sieve to remove any large particles such as shells which are found in the soil but which are not invariable constituents.

\section{Apparatus}

No detailed description of their dilatancy viscometer was given by Freundlich \& Röder, but it was described in detail by Röder (I939). A piece of apparatus was made which appeared to embody the essential features of the machine described. It is a device (Fig. 2) for pulling a sphere through the substance to be investigated. The soil is contained in a brass trough of square section $\mathrm{I} \cdot 6 \mathrm{~cm}$. deep and $50 \mathrm{~cm}$. long. Above the trough are arranged two brass guide rails, one vertically above the other, between which runs a small carriage. Fixed to this is a narrow shaft ending in a phosphor-bronze sphere of diameter $5 \mathrm{~mm}$. which can thereby travel along the locus of the long axis of the trough. The construction of the carriage is also shown in Fig. 2. It is attached by a thread, running over a pulley at one end of the guide rails, to a pan for holding weights. The pulley, carriage wheels and guide rails are of 


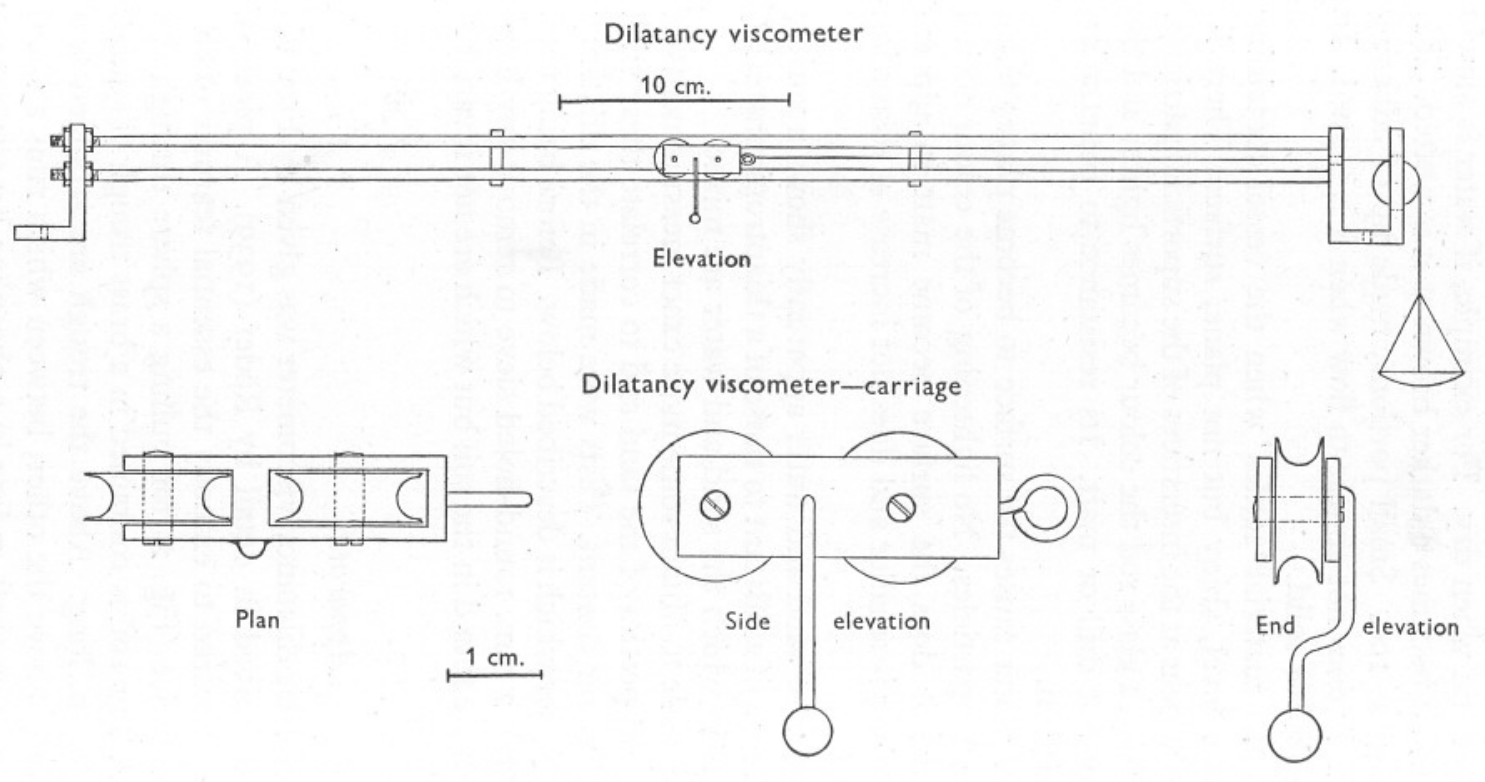

Dilatancy viscometer-trough

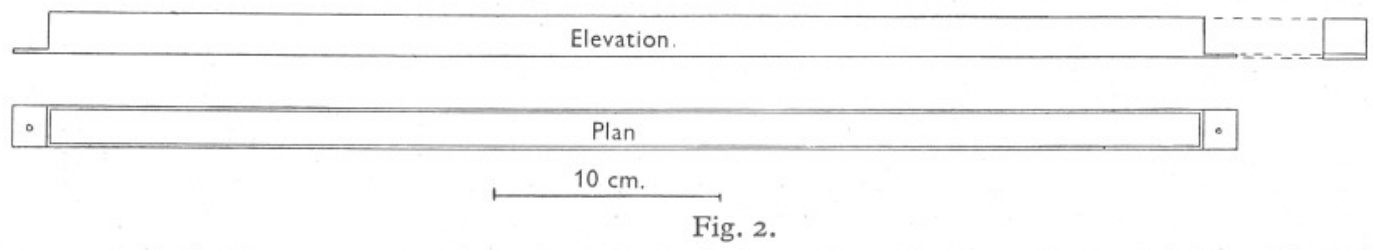


a high finish and are polished each time before use with household metal polish.

The time taken for the sphere to travel through a distance of $30 \mathrm{~cm}$. was measured by a stop-watch to the nearest $\frac{1}{5}$ th sec. Prior to making each run the soil was treated in a standard way, being tapped forty times on the bench and being left for $60 \mathrm{sec}$. before the test was begun. Even after using this standard preliminary treatment it was difficult to ensure that the soil was always in a 'natural' packing similar to that found in the sand of the undisturbed shore.

\section{Results}

The results obtained with this machine were plotted graphically as speed (ordinate) against weights placed in the pan. Ignoring the friction forces in the apparatus, the curve given by a Newtonian liquid (glycerol) is a straight line passing through the origin. According to Freundlich \& Röder a thixotropic substance also gives a straight-line graph, but instead of passing through the origin it cuts the abscissa some distance to the right. They obtained this result using a suspension of quartz particles in carbon tetrachloride, a nonpolar liquid, instead of water in which their quartz was dilatant. When once movement of the sphere begins in a thixotropic system its speed is proportional to weight as for a Newtonian liquid, but a minimum force is necessary before movement can begin, whereas with a perfect liquid the smallest applied force causes movement. (It would appear, however, that this 'ideal' curve for a thixotropic substance is not fully in accordance with the definition of thixotropy as decreased resistance to increased rate of shear.) Freundlich and Röder point out that dilatant substances do not give a straight-line graph but one which is a straight line only over the first part of the range of applied weights. Above a certain weight it flattens out so that no further increase in weight gives any increase in speed. In other words, when slow movement is taking place in dilatant systems - with small applied weights-their behaviour is nearly that of Newtonian liquids, but at a certain speed no further increase is possible without an incommensurate increase in applied weight. The form of the curves as they should appear according to Freundlich \& Röder is shown in Fig. 3. 'A horizontal part of the curve,' they say, 'implying solid behaviour, occurs in thixotropic systems at zero rate of shear, i.e. at rest....Liquid behaviour is represented in both cases by a steep part of the curve; in thixotropic systems at a sufficiently high rate of shear..., in dilatant systems at rest and at small rates of shear.'

Preliminary tests showed that the behaviour of the soil was complex. For example, in a mixture of sand and water with a small amount of superfluous water on top and with a weight of $20 \mathrm{~g}$. applied to the sphere, its speed through the soil was $0.05 \mathrm{~cm}$. $/ \mathrm{sec}$. on the first test after the trough was filled. After giving the trough forty taps and allowing the contents to settle for $60 \mathrm{sec}$, the speed rose to $3.0 \mathrm{~cm}$. $/ \mathrm{sec}$. After prodding the soil, as well as tapping it, the 
speed fell to $0.3 \mathrm{~cm}$. $/ \mathrm{sec}$., whereas on the fourth test, preceded only by the standard tapping procedure, the speed had risen to $0.6 \mathrm{~cm}$./sec. Further tests along these lines confirmed these results which showed that it was essential for the soil to be treated in a standard way before each test because the 'particle structure' of the soil was altered by the passage of the sphere, the alteration being at a maximum in rather dry sand, in which a clear channel was left. With dry sand the channel did not disappear even after the standard tapping procedure. The results of some of the preliminary tests, using sand saturated with sea water, are shown graphically in Fig. 4. It will be seen that there is no hint of a break in the curve such as Freundlich \& Röder say occurs with a dilatant material.

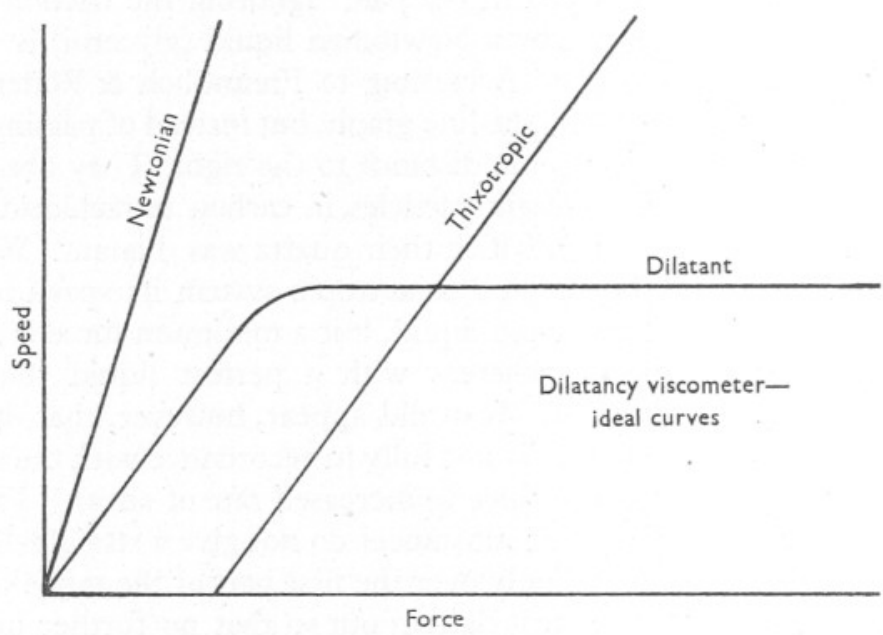

Fig. 3. Graph showing 'ideal' curves obtained by the use of the dilatancy viscometer.

The standard tapping procedure adopted may depend for its efficacy in standardizing the structure of the soil on the property described by Freundlich \& Jones as rheopexy. Suspensions showing this property solidify more quickly when subjected to moderate agitation.

\section{The effect of variations of water content}

If the soil behaved as a thixotropic substance when excess of water was present, might it not become dilatant when the water content was reduced? In order to test this possibility runs were made in the dilatancy viscometer using soil containing a different amount of water. The samples of sand and water were prepared by filling the trough with damp soil and adding sufficient water to cover it completely. Water was then drained off until the appearance of the soil was considered suitable. After the tests had been made the soil was scraped into a weighed beaker and dried in an oven at $105^{\circ} \mathrm{C}$. until of constant weight; 
from these weighings the water content was calculated. The results are given in Table I and are also shown plotted graphically in Fig. 5.

As can be seen from the table the results are not clear-cut. It is apparently true to say that the speed of the sphere is greater when the water content is

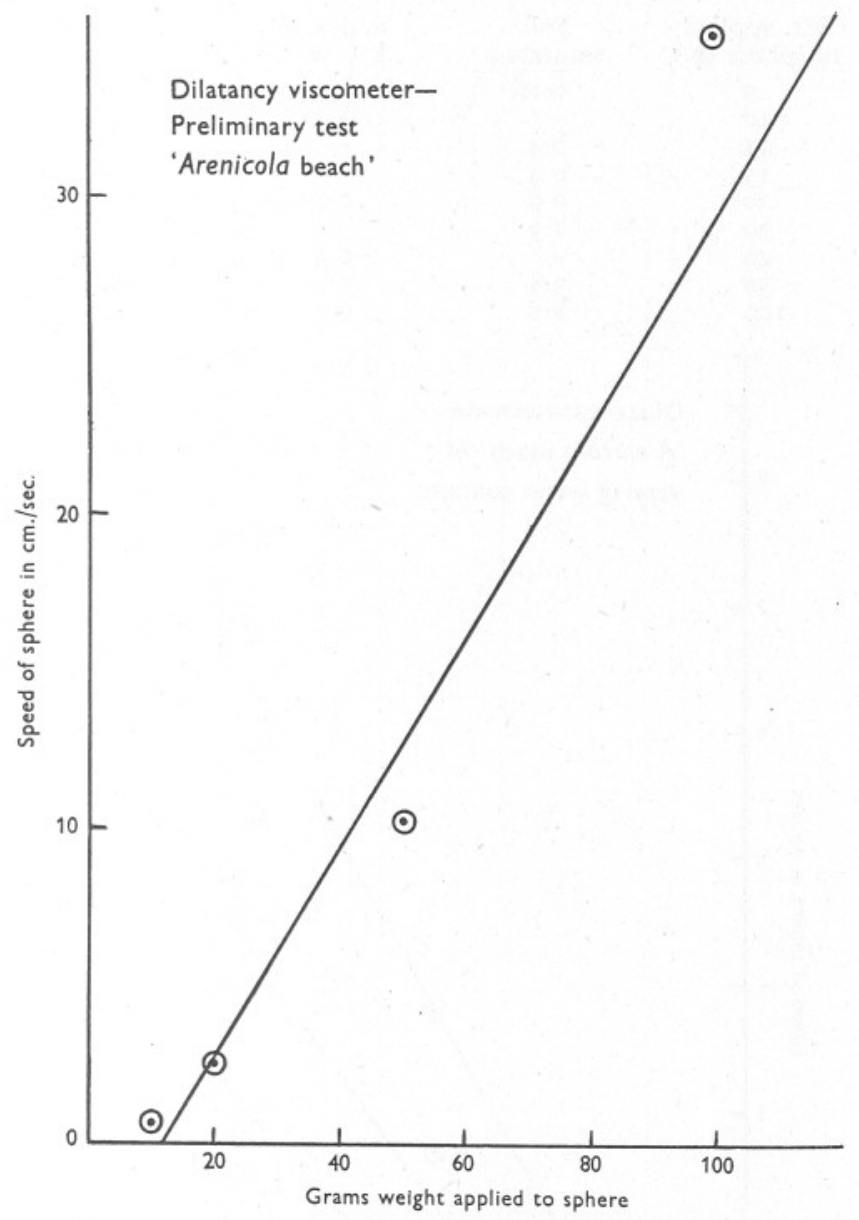

Fig. 4. Preliminary results of dilatancy viscometer tests.

higher, but over the range of weights applied the effects of dilatancy do not appear in the graphical result. The results agree with a thixotropic behaviour of the substratum in that the graphs cut the abscissa to the right of the origin. Against that, however, must be set the appearance of the sand. Especially when its water content is low its appearance is clearly of the dilatant type whilst the sphere is travelling through it. In front of the sphere the surface layer of the soil is shiny but not plane, the water surface being broken by sand 
Table I. The Effect of Water Content on the Speed of a Sphere Passing through Muddy Sand from the ARENicola Region of The Whitstable Flats

Speed of sphere in $\mathrm{cm}$./sec. Water content expressed as percentage wet weight of mixture. Almost all the figures for the speed of the sphere represent a mean of not less than three readings.

$\begin{array}{cccc}\begin{array}{c}\text { Wt. applied } \\ \text { to sphere (g.) }\end{array} & \begin{array}{c}\text { Soil } \\ \text { saturated }\end{array} & \begin{array}{c}\text { Soil with } \\ 28 \% \text { water }\end{array} & \begin{array}{c}\text { Soil with } \\ 21 \% \text { water }\end{array} \\ 9 & 0.16 & - & - \\ \text { I0 } & - & - & 0.04 \\ \text { II } & 0.4 & - & - \\ \text { I5 } & 0.9 & 0.25 & - \\ 20 & 0.9 & - & 0.15 \\ 30 & 1.5 & 0.9 & - \\ 40 & - & 2.7 & - \\ 50 & 3.8 & 6.0 & 1.25 \\ \text { I00 } & 8.6 & & 3.8\end{array}$

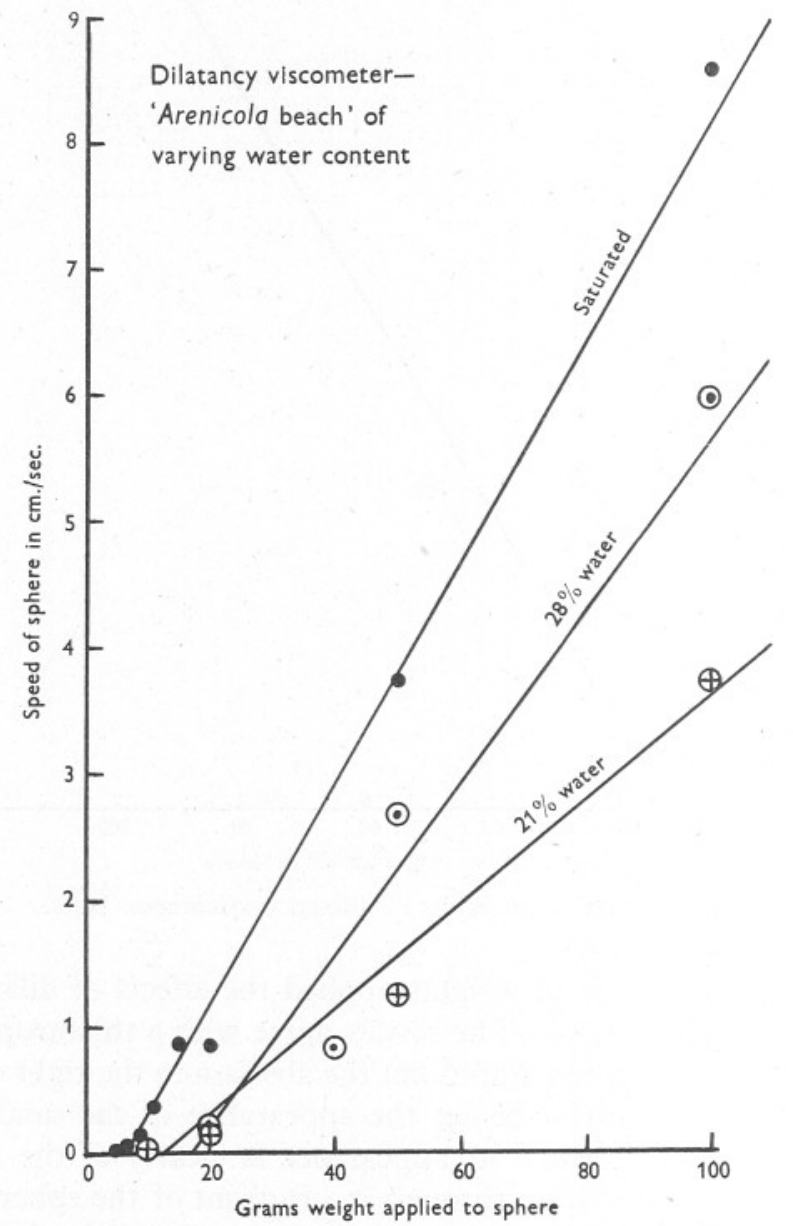

Fig. 5. Results of dilatancy viscometer tests on sand with varying water content. 
particles. Alongside and behind the sphere the surface appears dull or matt, the water now being insufficient to fill the enlarged interstices. This behaviour is characteristic of dilatant substances, as is the behaviour of the sand when the trough is being emptied. The sand becomes quite hard and firm so that it cannot be induced to flow but has to be scraped out.

It appears then as if the sand exhibits both dilatant and thixotropic properties in one sample and that, although its pore space may increase with the application of an external force, the resistance to shear does not reach a maximum at any particular rate of shear tested. It is possible, but unlikely, that this phenomenon was not shown over the range of forces which were applied. It is also possible that the amount of silt and clay present in the mixture was sufficient to render the mutual slipping of the particles easy enough to avoid any great increase in resistance to the applied shear force while yet not completely masking the fact that increase in pore space is effected by the shear force.

\section{The effect of the removal of silt and clay}

To see if a small amount of silt and clay might affect the physical properties of the soil, tests were made with the dilatancy viscometer, using sand which had been repeatedly washed in such a way that all the silt and clay were removed and all the fine sand left. This was done, as in the estimation of the various soil fractions by the method of Robinson, by the decantation of the supernatant liquid to a depth of $10 \mathrm{~cm}$. at a time of $4 \mathrm{~min} .48 \mathrm{sec}$. after the sample was dispersed by agitation. After repeated washings the sea water no longer appeared turbid and it was clear that all the silt and clay had been removed, unless there was any still adhering to the sand particles which could not be removed by washing but which might yet affect the physical properties.

Two tests were made with the washed sand, one in which there was a superfluity of water and the other in which the water content was found by subsequent analysis to be $2 \mathrm{I} \%$ by weight. The results are given in Table II and are plotted graphically in Fig. 6.

There was no very noticeable difference in behaviour between the washed and the unwashed sand during the course of the tests, and this was borne out by the graph which again showed that there was no clear indication, by a break in the straight line, of any marked dilatant behaviour of the sample of soil.

\section{Discussion}

Indeed, as Freundlich \& Röder remarked, the dilatancy viscometer is difficult to use with large particle systems, and it may be that therein lies the reason for the absence of any clear sign of dilatancy in the numerical results in spite of the clear signs of dilatancy given by qualitative tests. The only thing that does emerge clearly is that it takes longer for the ball to travel through drier sand than through wetter sand under a given weight, and that this is 
Table II. The Effect of Water Content on the SpeEd of a Sphere Passing through Washed, Silt-Free Sand from the Arenicola Region of the Whitstable Flats

Wt. applied

to sphere (g.)

IO

20

30

40

50

100

200
Soil saturated

0.3

I. O

I. 8

2.5

3.5

$7 \cdot 5$
$20 \cdot 0$
Soil with 2 I \% water

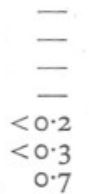

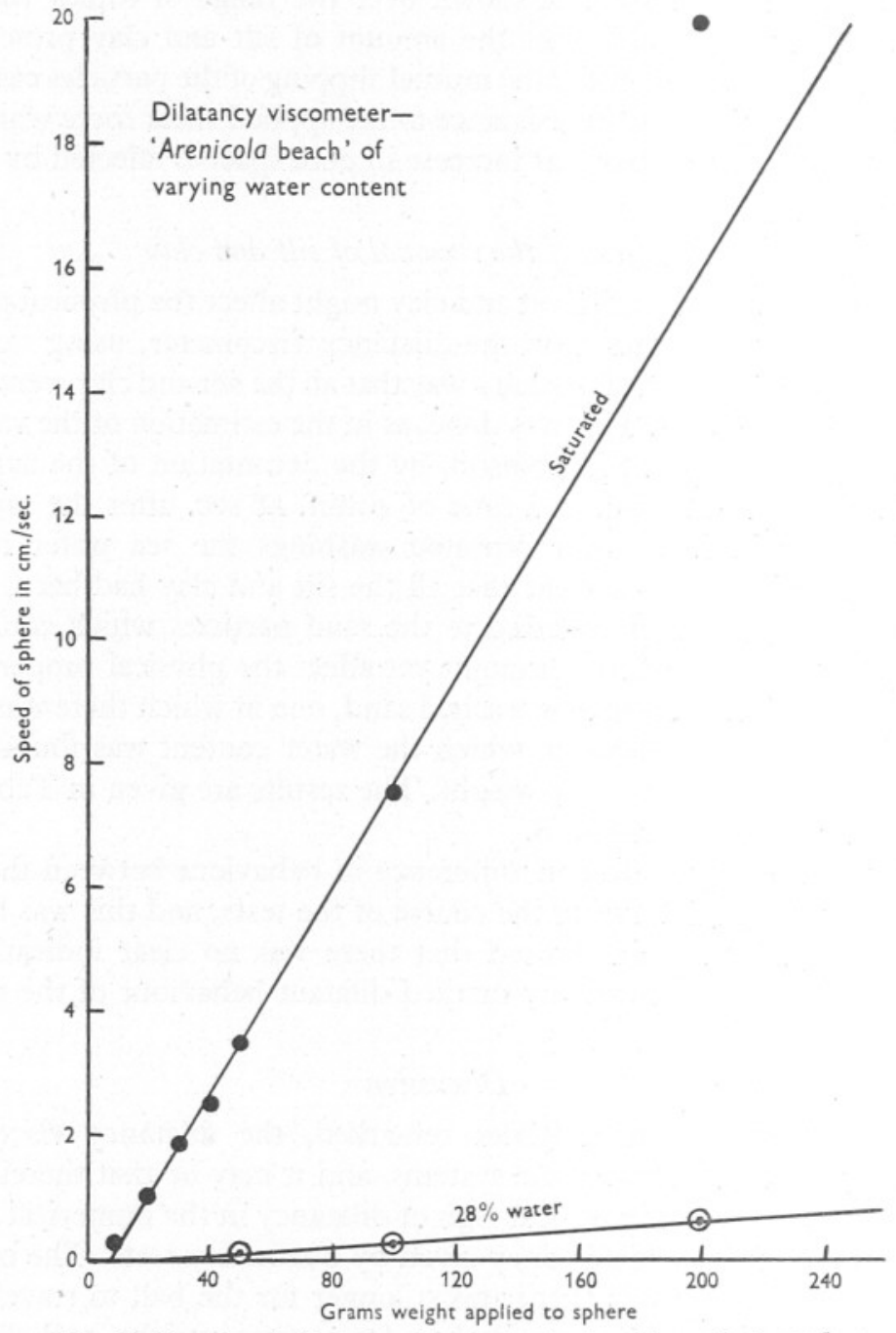

Fig. 6. Results of dilatancy viscometer tests with washed sand 
supported by the hardness measurements made with the penetrometer. Clearly the water content is important in the determination of the resistance of the sand to gross penetration and equally clearly the presence of excess water is necessary for the sand to become almost fluid as it may do when agitated. What is suggested, perhaps, by the straight-line graph of speed against force (which is characteristic of Newtonian liquids as well as of thixotropic substances) is that the limiting factor in the passage of the sphere through the system is the viscosity of the water. It can easily be seen in suspensions with only a small quantity of superfluous water above the sand that there is a visible flow of liquid from in front of to behind the sphere as it passes through the mass. If it can be shown that the sphere in its passage through the suspension leaves behind it a clear, fluid-filled channel (as it can be seen to do in relatively dry suspensions), then the liquid filling the channel has had to flow into its new position from elsewhere in the suspension. Clearly then, if there is a superfluity of liquid, more than enough completely to fill the interstices between the particles, liquid can flow in from all directions and will not have to come from very far away from the point at which the clear channel is being made. If, on the other hand, there is not a great superfluity of water, liquid will have to come from a greater distance away in order to fill the channel which is being made. Since it comes from a greater distance the time taken for it to accumulate will be greater and hence the speed of the sphere through the suspension slower.

If these suggestions are correct, then they would account for the straight-line graph obtained, because the relations of speed and force depend on the viscosity and density of the water which is a Newtonian liquid. Owing to turbulence of flow through the interstices between the particles the effect of viscosity is partly lost. But the hypothesis can be roughly checked by the substitution for water of other liquids with higher or lower viscosities and densities, the substitution of liquids with a higher viscosity and density should result in a slower rate of travel, and of liquids with a lower viscosity and density in a greater rate of travel for the same applied force.

Tests were accordingly made with dry sand to which was added one of the three polar liquids glycerol, water and ether, the viscosities of which are in the approximate ratio of 5000:5:I and the densities in the approximate ratio of $I \cdot 6: I \cdot 3: I$. With the same weight in the scale-pan the speed of the sphere through sand-glycerol, sand-water and sand-ether mixtures was in the approximate ratio of 50:5:I. Had the viscosity factor alone been operative the ratio of speeds should have approached more nearly to 5000:5:I, and had the density factor alone been operative the ratio of speeds should have been nearer to I.6:I·3:I. In a general way the results of this test confirm the suggestion that the viscosity and density of the interstitial liquid may be one of the main factors influencing the movement of marine soil. It is not suggested that this has been proved but merely that the absence of any kind of dilatant behaviour 
revealed by the viscometer and the rough correspondence of speeds and viscosities and densities suggests that the physical properties of the liquid determine the behaviour of marine soils as well as the size of the component particles.

\section{SoIl Hardness and Burrowing SpeEd of ARENICOLA}

Since the purely physical method of the dilatancy viscometer did not show clearly any very striking difference between washed and unwashed sand, or between saturated and drier sand, a biological method was tried for testing the properties of the soil. The speed of burrowing of Arenicola marina L. was used for this purpose, since a certain amount is already known about this activity (Chapman \& Newell, 1947). The usefulness of the animal as a biological tool is due to the fixity of its behaviour. When placed upon the surface of the substratum it at once begins to burrow in, whether its ventral or its dorsal surface is in contact with the soil. It seldom attempts to 'right itself' first. If the first attempt with its proboscis should fail to find a suitable spot it may try other places, but equally, it may persist for some time in attempting to force its way into an unsuitable place. Its behaviour seems quite unchanging although the environment may vary. This fixity does not leave scope for any kind of crawling or seeking behaviour. Hence, when the creature is placed on a soil into which it is not prevented from burrowing by large stones, the time which it takes to enter the soil, from the instant at which it is placed on the surface, can be taken as an indication of the difficulty which it has in the penetration of the soil.

The time taken by samples of worms to burrow into sands of different water content was measured and the sands subsequently sampled and analysed. The sampling was done with a cork borer which removed, with very little disturbance, a cylinder of soil of $\mathrm{I} \mathrm{cm}$. diameter; the analysis was done by drying a sample of some $15 \mathrm{~g}$. to constant weight at $105^{\circ} \mathrm{C}$. The samples of sand of different water content, in which the exercises were carried out, were arranged by making small mounds of different heights on the Flats. Water then drained from the upper parts of these leaving them drier than the surrounding shore. Alternatively, a circular moat was dug round a central 'island' which again became drier by the draining of water into the moat. This latter method had the advantage of not disturbing the natural structure of the sand. But even in the other method an attempt was made to preserve this structure as far as possible by being careful to construct the mound of clean spits of sand, in which the natural upper surface was left uppermost.

In the first set of experiments three batches, each of ten worms, were used. The first batch constituted a control and were allowed to burrow into undisturbed sand, the second batch were set to burrow into a low mound and the third batch into a higher mound. In the first set of experiments the water 
content of the mounds was not estimated. A second set of experiments was made in a similar fashion and the water content of the soil was estimated. A selection of the detailed results of these tests is set out in Table III. They are quite clear-cut and from them it can be seen that the worms were quite unable to burrow into the high mound from which the water appeared to have drained. Some of them could burrow into the low mound, but those which succeeded in doing this took longer than the worms which burrowed into the undisturbed sand. Worms which had been trying to burrow into the high mound were put on to the surface of undisturbed beach, into which they burrowed at about the normal rate.

Although the weighing error in estimating the water content of the sand samples did not exceed $\pm 0.1 \%$ it would seem that the accuracy of sampling, even if not the accuracy of estimation of the water content, was not sufficiently accurate to yield useful results. It may be that the method of taking the sample, disturbing the sand as little as it did, was yet unsuitable for the estimation of very small differences in the water content. It was, however, very striking that the animal was quite unable to cope with the drier sand which, of course; was distinctly harder to feel and apparently dilatant when disturbed. It would appear that another method of estimating the effect which diminution of its water content had on the sand was necessary, so that hardness tests were made with the penetrometer.

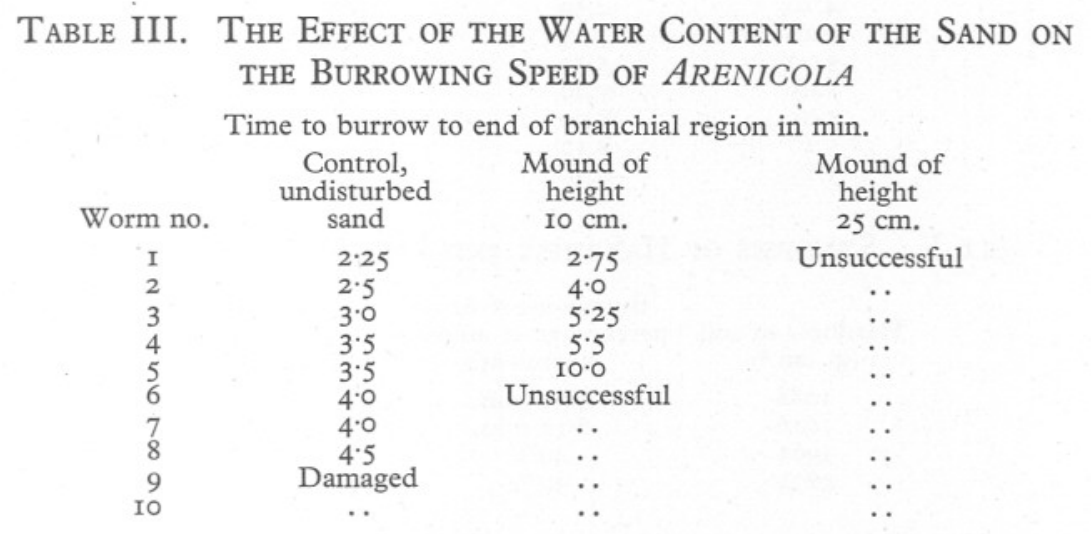

After ro min. the worms from the high mound were placed on undisturbed sand when they burrowed in the following times: $2 \cdot 5,2 \cdot 75,3 \cdot 5,4 \cdot 0,4 \cdot 25,4 \cdot 5,5 \cdot 0,5 \cdot 25,5 \cdot 5,5 \cdot 75$.

In the third set of experiments the same methods as before were used to produce small areas of soil in which the water content differed from that of the surrounding shore, but the water content of the soil was not estimated. Instead the hardness of the soil was measured with the penetrometer. The results are given in Table IV from which it can be seen that they agree with the first two sets of tests. As the height of the mound increases so the 
hardness of the soil increases and the ability of the worms to burrow into it decreases.

A synopsis of the experiments on the correlation of soil hardness and burrowing times is set out in Table V.

\section{CONCLUSIONS}

The aim of the work which has been set out in this paper is ultimately to determine to what extent the physical properties of the substratum determine the kind and number of its inhabitants, particularly in the intertidal zone. It is well known that some worms prefer fine soil, others a coarser medium, but it

\section{Table IV. The Effect of Water Content on the Hardness of the SoIl and the Burrowing SPEed of ARENICOLA}

Burrows made into mounds of varying height above water level. Time to burrow in min. and sec. Hardness in $\mathrm{g} . / \mathrm{cm} .{ }^{2}$ required for penetration. (Each figure represents a mean of not less than twelve readings.)

\begin{tabular}{|c|c|c|c|c|}
\hline Worm no. & $\begin{array}{c}\text { Mound height } \\
\text { zero. } \\
\text { Hardness } \\
\text { I088 }\end{array}$ & $\begin{array}{c}\text { Mound height } \\
5 \mathrm{~cm} \text {. } \\
\text { Hardness } \\
\text { 1616 }\end{array}$ & $\begin{array}{c}\text { Mound height } \\
7.5 \mathrm{~cm} . \\
\text { Hardness } \\
\text { I96I }\end{array}$ & $\begin{array}{c}\text { Mound height } \\
\text { I3 cm. } \\
\text { Hardness } \\
2272\end{array}$ \\
\hline I & 2.30 & 4.20 & 8.00 & 6.00 \\
\hline 2 & 3.15 & $4 \cdot 35$ & $16.00+$ & 10.00 \\
\hline 3 & 4.00 & 4.45 & $16.00+$ & 10.00 \\
\hline 4 & 4.10 & 4.50 & $16.00+$ & Unsuccessful \\
\hline 5 & 4.20 & 5.15 & Unsuccessful & $\ldots$ \\
\hline 6 & 5.00 & 5.50 & $\ldots$ & .. \\
\hline & 5.05 & 7.20 & .. & .. \\
\hline 8 & 5.05 & 8.00 & .. & . \\
\hline 9 & 5.10 & 8.10 & .. & .. \\
\hline Io & 5.15 & 8.30 & .. & .. \\
\hline
\end{tabular}

TABLE V. Synopsis of Hardness and BurRowing Experiments

$\begin{array}{ccc}\begin{array}{c}\text { Hardness of soil } \\ \left(\mathrm{g} . / \mathrm{cm} .{ }^{2}\right)\end{array} & \begin{array}{c}\text { Burrowing time or } \\ \text { percentage of animals } \\ \text { burrowing }\end{array} & \begin{array}{c}\text { Height of } \\ \text { mound }(\mathrm{cm} .)\end{array} \\ \text { 1088 } & 4.38 \mathrm{~min} . & \text { Zero } \\ \text { 1616 } & 6.17 \mathrm{~min} . & 5.0 \\ \text { 1961 } & 40 \% & 7.5 \\ 2272 & 30 \% & 13.0\end{array}$

is not known precisely how these changes in composition affect the properties of the soil which are important to the animals. In the intertidal zone, from which the soil used in the experiments was obtained, even an apparently uniform environment may be subject to small local variations of silt or water content which are sufficient to bring about marked changes in the properties of the soil. The Flats at Whitstable are uncovered for about Io out of the $24 \mathrm{hr}$., so that the time during which the animals may be subjected to varying physical conditions is an appreciable part of their life. The slope of the shore, which is 
here very gradual; the nature of the underlying stratum, whether porous or impervious; small local variations in elevation; shelter from wind or sea scour; all these may serve to alter the physical properties which have been studied.

An attempt has been made to show that the properties of thixotropy and dilatancy, which are of importance to burrowing animals, are affected by water content, that the hardness of the soil varies with water content and that the burrowing speed of lugworms is also affected. It is disappointing to find that the physical method of investigation using the dilatancy viscometer of Freundlich \& Röder did not give clear-cut results, and did not show that there was any marked difference in properties between wet and dry sands, nor between those containing silt and clay and those which had been washed free from small particles. But the results obtained from the machine suggest that it is the density, viscosity and amount of water which determines to a large extent the fluidity of the sand on agitation. This conclusion was supported by tests which were made using liquids having viscosities and densities different from that of water.

Changes in the amount of water mixed with the sand of the Flats caused a marked change in its hardness and the speed with which lugworms could burrow into it. This is clearly a property which may be of great importance in the distribution of Arenicola. At Whitstable the sand is underlain by impervious clay and does not dry out at low water. It is possible that the worms can 'work' the headshafts of their burrows (Wells, 1945) at all states of the tide. But if there were no clay to retain the water, then at low tide the drained soil would become too hard for them to 'work', their feeding time would be limited to the time during which they were covered by the water and their distribution might well be limited also. The Flats appear too uniform in water content and worm population for the correlation of these features to be very striking, and it is hoped that some more suitable site will be investigated. But it is clear that there are considerable differences in the density of population of small local areas, the arrangement of the burrows in straight lines being one of the most striking (Newell \& Chapman, 1948).

The effect of the clay content on the physical properties of the soil, for which it was not easy to obtain numerical data from the dilatancy viscometer, may also prove more susceptible of investigation by a biological method. The burrowing speed of Arenicola which has been correlated with the hardness of the soils would clearly be suitable for this purpose.

\section{SUMMARY}

Modifications are described to the penetrometer originally designed by Chapman \& Newell (1947) for the measurement of the resistance to penetration of marine soils. 
An account is given of qualitative and quantitative tests made during the study of the thixotropy and dilatancy of the muddy sand of the Arenicola region of the shore at Whitstable. The results of tests made with the dilatancy viscometer originally used by Freundlich \& Röder are shown not to be in clear agreement with the observed behaviour of the soil.

It is suggested that the fluidity or otherwise of a marine soil is a function of the viscosity and density of the liquid filling the interstices between the particles.

It is shown that the resistance to penetration of the soil and the burrowing speed of Arenicola are dependent upon the water content.

\section{REFERENCES}

Boswell, P. G. H., I948. The thixotropy of certain sedimentary rocks. Science Progress, No. I43, pp. 4I2-22.

Chapman, G. \& Newell, G. E., I947. The role of the body-fluid in relation to movement in soft-bodied invertebrates. I. The burrowing of Arenicola. Proc. Roy. Soc., B, Vol. cxxxiv, pp. 43I-55.

Freundlich, H., I935. Thixotropy. Paris: Hermann.

Freundlich, H. \& JoNES, A. D., I936. Sedimentation volume, dilatancy, thixotropy and plastic properties of concentrated suspensions. Fourn. Phys. Chem., Vol. XL, pp. I $217-36$.

FreUndlich, H. \& JUliusburger, F., I935. Quicksand as a thixotropic system. Trans. Faraday Soc., Vol. xxxI, pp. 769-74.

FREUNDLICH, H. \& RöDER, H. L., I938. Dilatancy and its relation to thixotropy. Trans. Faraday Soc., Vol. xxxiv, pp. 308-I6.

KruYt, H. R. \& VAN Selms, F. G., I943a. The influence of traces of water on the plasticity of starch and quartz suspensions in organic media. Rec. Trav. Chim. Pays-Bas, Vol. Lxir, pp. 407-I4.

- $1943 b$. The influence of a third phase on the rheology of suspensions. Rec. Trav. Chim. Pays-Bas, Vol. Lxir, pp. 415-26.

Newell, G. E. \& Chapman, G., I948. Distribution of lugworms. Nature, Vol. CLXII, p. 75 .

Pryce-Jones, J., I946. The flow of suspensions-thixotropy and dilatancy. Proc. Univ. Durham Philos. Soc., Vol. x, pp. 427-67.

REYNOLDS, O., I885. On the dilatancy of media composed of rigid particles in contact. With experimental illustrations. Phil. Mag., Vol. xx, pp. 469-8I.

RobInson, G. W., I932. Soils, their Origin, Constitution and Classification. London. RöDER, H. L., I939. Rheology of Suspensions. Amsterdam: Paris.

VAN Selms, F. G. \& KRUYT, H. R., I943. Apparatus for measuring the consistency of plastic suspensions. Rec. Trav. Chim. Pays-Bas, Vol. LxII, pp. 398-406.

Wells, G. P., I945. The mode of life of Arenicola marina L. Fourn. Mar. Biol. Assoc., Vol. xxvI, pp. I70-207. 